\title{
La generalización en cartografía básica y temática
} Generalization in basic and thematic Cartography

Fecha de recepción: 30 de marzo de 2007

Fecha de aceptación: 13 de julio de 2007

María Fernanda Galvis Lagos*

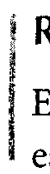

\section{RESUMEN}

El conocimiento y explicación de la organización espacial y sus variaciones es inherente a la Geografía y constituye su esencia para la expresión gráfica (cartográfica).La organización espacial puede entenderse como una jerarquía encajonada de sistemas y subsistemas a diferentes escalas; por tanto requiere ser clasificada jerárquicamente y representada cartográficamente como espacios geográficos de diversos tamaños, dando cabida a la generalización cartográfica. Sin embargo, al revisar los materiales cartográficos producidos por diversas entidades nacionales es evidente el uso de clasificaciones planas o no jerarquizadas (a un único nivel). Este hecho obstaculiza el proceso de generalización cartográfica y presenta serias dificultades tanto conceptuales como de procedimiento en el momento de cambiar la escala cartográfica. En este trabajo se plantea una conceptualización de generalización cartográfica con base en la teoría jerárquica y la noción de nivel de resolución, y se muestra cómo la vocación aplicada o instrümental de la clasificación jerárquica es la cartográfica, puesto que cada nivel jerárquico necesita una dimensión espacial suficiente para expresarse.

* Ingeniera Catastral y Geodesta, Universidad Distrital Franciscó José de Caldas. Especialista en Sistemas de Información Geográfica, Universidad Distrital Francisco José de Caldas; especialista en Matemáticas y Estadistica Aplicadas, Universidad Pedagógica y Tecnológica de Colombia; magister en Geografia con énfasis en Ordenamiento Territorial,-Universidad Pédagógica y Técnológica de Colombia.

Docente Universidad Distrital Francisco José de Caldas (código 3í50). 
Definida la base conceptual, se propone un procedimiento de generalización cartográfica basado en un modelo jerárquico escalar, que define los conceptos que se desarrollan en cada nivel de la jerarquía y los rangos escalares en los que se representa cada nivel. Además se muestra el empleo correcto de las variables visuales que reflejan el nivel de la información y la clasificación jerárquica. La propuesta anterior se aplica en seis ejemplos de generalización para cartografía temática, en los cuales la clasificación jerárquica es fundamental para, a partir del nivel de mayor resolución, avanzar hacia niveles jerárquicos superiores que se representan en escalas cada vez menores.

\section{Palabras clave}

Generalización, cartografía, generalización conceptual, generalización estructural, nivel de resolución, escala, holon, jerarquía, organización jerárquica, clasificación jerárquica.

\section{ABSTRACT}

The knowledge and explanation of the spatial organization and its variations is inherent to Geography and constitutes its essence for cartographic expression. Spatial organization can be understood as different scale systems and subsystems boxed hierarchy for which it must be hierarchically classified and represented cartographically in geographic spaces of different sizes giving rise to cartographic generalization. However, by revising cartographic materials produced by different national organizations it is evident that the use of flat classifications obstructs the cartographic generalization process and shows serious concept and procedure difficulties when the cartographic scale is changed. In this paper, therefore, a cartographic generalization concept based on the hierarchical theory and the resolution level notion is outlined, and it is shown how the applied or instrumental vocation of hierarchical classification is the cartographic one, since each hierarchical level needs an enough spatial dimension in order to express itself. Once conceptual basis is defined, a cartographic generalization procedure based on a scalar hierarchical model is posed, that defines the concepts developed in each hierarchy level and the scalar ranks on which each level is represented. Moreover, the correct use of visual variables that reflect information level and hierarchical classification is shown. The preceding proposal is applied in six examples of generalization 
for thematic cartography, in which, beginning with the higher resolution level, hierarchic classification is basic for making advances tohierarchically higher levels that are represented at smaller scales.

\section{Key words}

Generalization, Cartography, concept generalization, structural generalization, resolution level, scale, holon, hierarchy, hierarchical organization, hierarchical classification.

\section{INTRODUCCIÓN}

Uno de los conceptos centrales que se maneja en la geografía es la escala, entendida como una de las condiciones gráficas de representación que corresponde a un nivel de resolución. El mapa es la expresión o retrato de la superficie terrestre, por tanto de los hechos o fenómenos geográficos que permiten conocer, entender, describir o explicar la localización y evolución de los recursos biofísicos, ambientales, humanos. Es decir, una cantidad enorme de información espacial que si no se discrimina y se generaliza en un nivel de resolución legible sería incomprensible. Puesto que todo mapa es una representación generalizada de características geográficas, en él se sintetizan las particularidades espaciales para hacerlas observables Por consiguiente, el tratamiento geográfico de lo real está afectado por diferentes niveles de resolución que se manifiestan en diversas escalas y que se traducen en múltiples comprensiones de los fenómenos.

Como nada se explica completamente en un nivel de resolución homogéneo, surge la necesidad de proporcionar múltiples perspectivas de fenómenos géográficos con diferentes nivelés dé resolución que representen los distintos modos de percepción y concepción de lo real, y definan la escala que permite hacer visible el fenómeno y posibilita su medición, ánálisis y explicación. En cartografía el único proceso válido és disminuir la escala; por esto, el término generalización cartográfica se aplica a la manipulación deliberada de la información geográfica para crear un mapa derivado a partir de un mapa que está a una escala mayor y con un mayor nivel de resolución.

Aunque en países con mayor nivel científico y tecnológico el proceso de la generalización cartográfica se ha tratado desde hace tiempo, en Colombia 
es muy escaso el conocimiento construido y aplicado, y los avances del tema han llegado más por la adquisición de tecnología. Lo anterior se manifiesta en los frecuentes errores del manejo de cartografía por carecer de conceptos claros al respecto.

En el manejo de la información geográfica se encuentran errores relacionados con cambios indiscriminados en la escala y en las categorías conceptuales que en cada caso se incluyen. Al analizar la cartografía que manipulan diversas entidades, por ejemplo para ordenamiento territorial, se percibe que se toma un mapa a una escala determinada y se realizan ampliaciones o reducciones sin tener en cuenta la relación entre el contenido y el tamaño de la representación. Es importante tener en cuenta que esto sugiere problemas en el nivel de análisis o síntesis, el nivel de concepción y el nivel de representación cartográfica, puesto que están limitados y sesgados por la escala cartográfica que representa el fenómeno. De esta forma, se niega la posibilidad de percibir lo que pasa a escalas superiores o inferiores, afectando significativamente el análisis y la explicación del fenómeno.

Además de los problemas mencionados, en la cartografía temática no se emplea una estructura de clasificación de información en la que se aprecie un manejo conceptual de niveles de resolución, pues no se tiene en cuenta que la generalización obedece a un cambio en los objetivos y está conceptualmente ligada a un cambio en el nivel de resolución; en otras palabras, se carece de formación para elaborar clasificaciones jerarquizadas que permitan la generalización.

Dado lo anterior, los objetivos propuestos son: examinar los niveles de resolución de las representaciones espaciales para orientar el proceso de generalización cartográfica; proponer una conceptualización y metodología de generalización cartográfica desde la geografía y técnicas cartográficas; orientar la definición de clasificaciones jerárquicas según niveles de resolución en la representación cartográfica de las organizaciones espaciales; y desarrollar procedimientos y mostrar aplicaciones de generalización en cartografía temática a partir de trabajos existentes, previa adecuación de la información. 


\section{CONCEPTOS BÁSICOS}

\section{Generalización cartográfica}

Sólo en escalas y resoluciones más grandes, la representación puede ser más aproximada a la realidad. La elaboración de un mapa requiere especificaciones técnicas que dependen de la escala. Así, el mapa a escala grande tiene mayor exigencia de precisión y exactitud que uno de escala pequeña. Por esto, en los procedimientos cartográficos, sólo es posible generalizar, es decir, disminuir la escala y el nivel de resolución.

Al generalizar se pasa de un nivel de resolución detallado a uno más general o menos detallado. Como se observa, los límites de resolución de los mapas están conectados a la escala y a la habilidad para representar ciertos rasgos en un mapa. Más allá de estos límites los rasgos deben ser generalizados, desplazados o abstraídos para permitir un mapa legible y estéticamente agradable. Además, en la cartografía temática, al generalizar es importante tener en cuenta las variables visuales puesto que puede cambiar el nivel de la información y la forma de implantación.

La generalización cartográfica es motivada por la necesidad de proporcionar escalas más pequeñas y con menores niveles de resolución de los datos geográficos que permitan visualizar áreas mayores con un puntọ de vista más general.

\section{Generalización estructural}

La generalización estructural se refiere a la reducción de la cantidad de información cuando se reduce la escala de un mapa, asegurando que la información sea legible para el usuario del mapa. Sobre los mapas topográficos se realiza este tipo de generalización, que puede ser concebida "como un filtro que remueve el ruido de una señal para realzarla", como lo señala Skarstein (1980: 99), sobre todo mediante procesos de selección, simplificación, combinación, exageración y desplazamiento. Sin embargo, la generalización estructural en mapas topográficos no es muy flexible. Flórez y Montoya (1992: 86) señalan como requisitos para la generalización de cartografía básica: mantener la planimetría, conservar la forma y las características de los accidentes, y tener en cuenta la relación entre los elementos del mapa. En la generalización estructural se simplifica la distribución de los elementos, pero se conserva la conceptualización y la estructura del fenómeno. 


\section{Generalización conceptual}

La generalización conceptual se produce cuando hay un cambio conceptual en el mapa, es decir, no sólo varía la escala sino que también varían los objetivos del mapa, por lo cual el fenómeno requiere otro tipo de expresión gráfica, que generalmente se expresa en un cambio de implantación. La Asociación Cartográfica Internacional (International Cartographic Association, ICA, 1973) señala que "la generalización conceptual transforma el modo de representación de un fenómeno para responder a un nuevo concepto y/o a un cambio en el nivel de observación".

Al generalizar puede cambiar la estructura y el concepto que se emplea pára referirse al objeto. Por ejemplo, de un municipio se puede expresar el concepto de extensión, en un nivel de detalle y a una escala que lo permita, pero al generalizar se llega a una escala en la que ya no es posible manejar el concepto de extensión. Así, la información que se proporciona del objeto es otra y se debe cambiar el concepto de extensión por el concepto de ubicación en el máximo nivel de abstracción. Si al generalizar, desaparece un concepto, también desaparece cualquier forma de representación cartográfica,

\section{Holon}

Estudiar el universo como una serie de sistemas, unos dentro de otros, no es un ejercicio conceptual fácil. Según lo explica Haigh (1987), la teoría de sistemas es válida para estudiar las interacciones entre los sistemas y para estudiar los sistemas mismos. Un sistema es un conjunto estructurado de interacciones reconocidas para el propósito de la investigación. Cada sistema natural existe como un todo en sí y como parte de otro todo más amplio.

El concepto clave en la teoría de sistemas es la noción de holon. Koestler acuñó el término holon (del griego holos, "todo", junto con el sufijo on, "parte") para referirse a aquello que, siendo una totalidad en un contexto, es simultáneamente una parte en otro contexto. Un holon, explica Haigh (1987), es cualquier subconjunto estable en una jerarquía, que se caracteriza por dos aspectos: primero, sus interrelaciones con el conjunto de orden mayor en el cual se integra y por el que sus actividades son limitadas; segundo, es un todo en su derecho propio, lo que integra las operaciones de los subconjuntos de nivel inferior. La ubicación de los holons en la jerarquía está ligada a la escala 
en cartografía, siendo las escalas de los niveles superiores las espacialmente más groseras.

\section{Nivel de resolución}

Un concepto primordial en la teoría de sistemas complejos y en la cartografía es el nivel de resolución. Chadwick (1981) comenta que a medida que aumenta el nivel de resolución se tendrá un sistema con muchos holons y relaciones complejas, y si se diminuye el nivel de resolución sè pierde significancia individual. El nivel de resolución está ligado al requerimiento de detalle con que se define el sistema. En función del interés con que se percibe ún sistema, se define el nivel de conocimiento de los objetos y de la globalidad del sistema.

La escala está en relación directa con el nivel de resolución: un nivel de alta resolución requiere una escala grande para su representación cartógráfica; si el nivel de resolución disminuye, es decir, se representa un concepto más general, se requiere una escala más pequeña (figura 1 ).

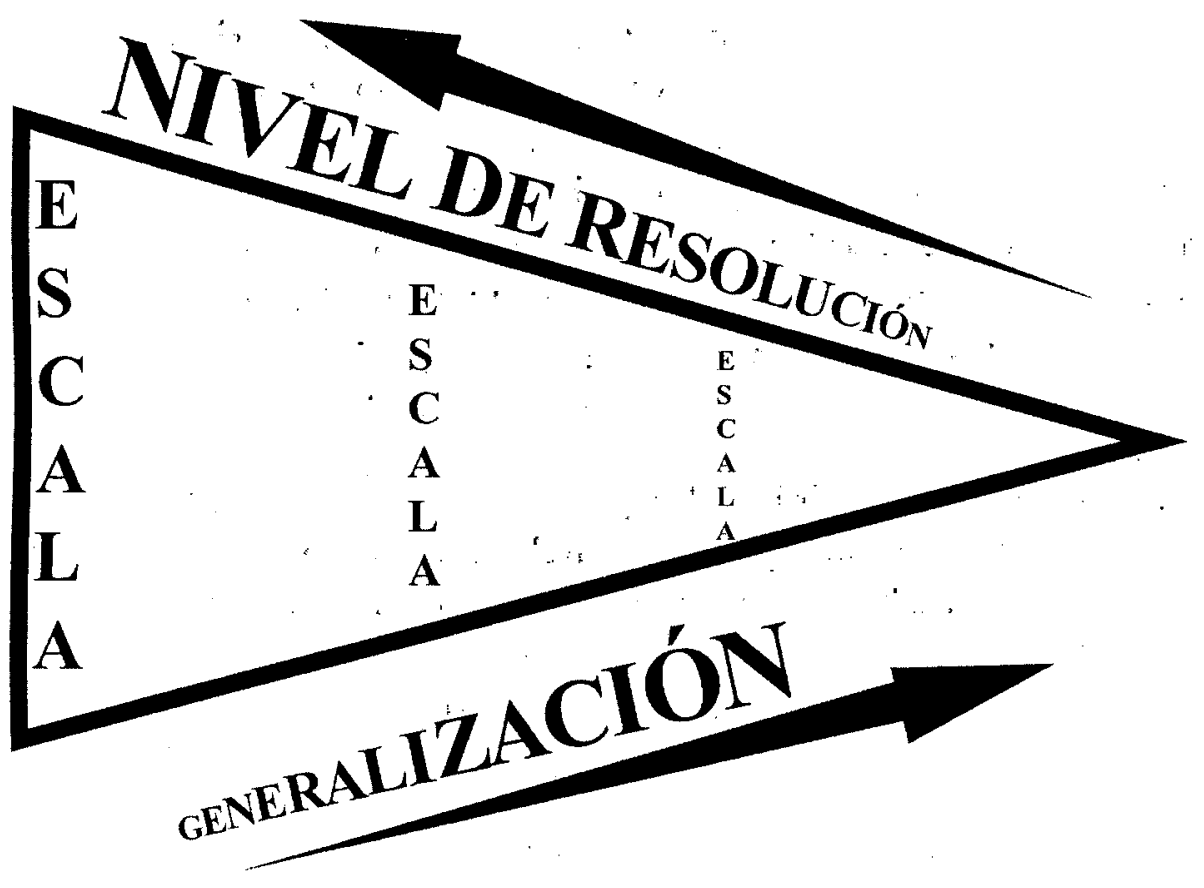

Figura1 Nivel de reșolución y generalización. 


\section{Clasificación jerárquica}

Para Haigh (1987), la estructura jerárquica es una propiedad fundamental de todos los sistemas naturales. Ibáñez (2006) señala que una de las principales premisas de la teoría de sistemas se basa en la idea de que, en el estudió de los sistemas jerárquicos, la comprensión de un nivel de la jerarquía requiere el conocimiento adecuado de los niveles inmediatamente superior e inferior.

La organización jerárquica se refiere al arreglo de las relaciones entre los holons que producen un sistema complejo y determina internamente dos manifestaciones contrarias: las emergencias y los constreñimientos. Las emergencias son propiedades y características de los holons de nivel superior, de las cuales carecen los holons de nivel inmediatamente inferior, considerados de forma aislada o dispuestos en otro sistema. Los constreñimientos son propiedades y cualidades que pierden los holons de nivel inferior al formar un holon de nivel superior.

En la clasificación jerárquica cada nivel permite un grado de abstracción que será menor en el último nivel y mayor en el primer nivel. Por tanto, se requerirá la escala más grande para presentar el último nivel de la clasificación y se representarán en escalas cada vez más pequeñas los niveles superiores hasta llegar al máximo nivel de generalización conceptual. En consecuencia, al clasificar de manera plana (un solo nivel) y representar fenómenos en una única escala, se limita la capacidad de pensamiento. Este desatino se corrige con la taxonomía (clasificación jerárquica), que permite la representación cartográfica en tantas escalas como niveles tenga la jerarquía.

La clasificación jerárquica ayuda a pensar en la complejidad del fenómeno estudiado, pues permite captarlo con niveles de resolución opuestos, general y detallado, pero a la vez complementarios. Los diferentes niveles en la clasificación jerárquica son a la vez productos y productores; además, cada nivel manifiesta aquello que es todo y parte a la vez (holon), ofreciendo la posibilidad de concebir el todo y las partes, asociados a niveles de resolución.

La clasificación jerárquica se puede considerar como una división o taxonomía vertical de los objetos, puesto que toma en cuenta su jerarquía y su subordinación, por lo cual, crea posibilidades de clasificación en muchos 
niveles. Un sistema de clasificación como éste, con dos o más niveles, permite generalizar, universalizar, llegar a un mayor nivel de abstracción.

Una vez organizada la información en los diferentes niveles de la jerarquía, se puede generalizar (disminuir la escala al pasar del nivel inferior a los niveles superiores) puesto que para algunos propósitos ciertos holons de niveles inferiores o subsistemas se tratan como "cajas negras" (o como el nivel inferior del sistema a considerar). En otras palabras, para ganar nueva información a un nivel más alto se requiere ocultar o suprimir los niveles más bajos. Sin embargo, como se ha manifestado, se requiere más información para generar un nivel de información más detallado (figura 2). .

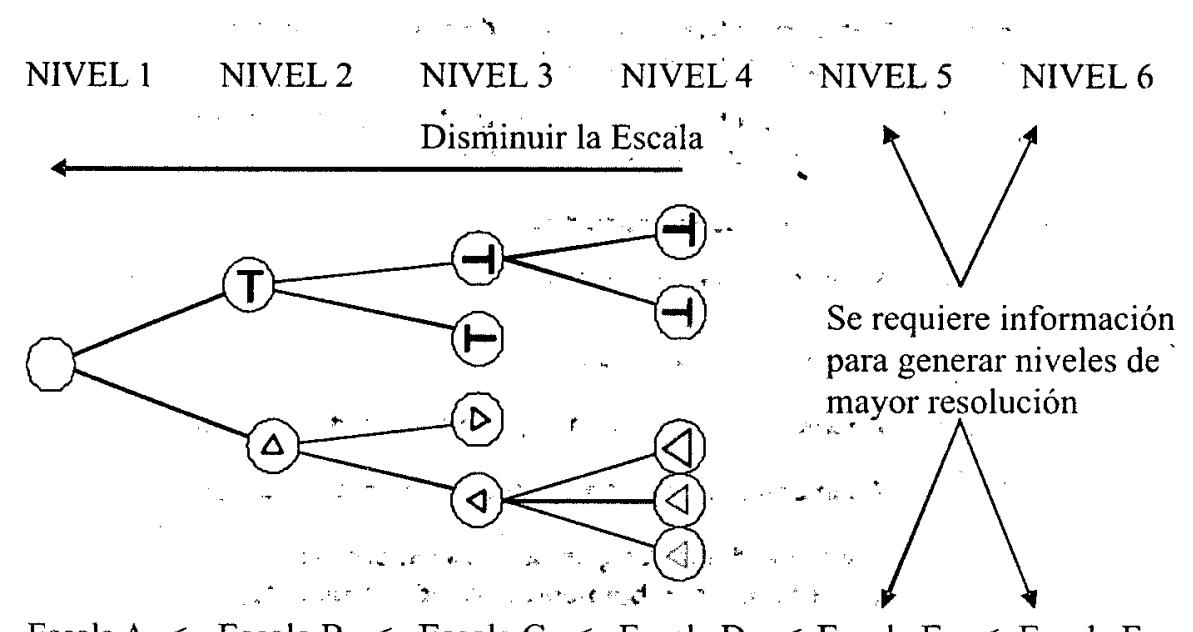

Escala $\mathrm{A}<$ Escala $\mathrm{B}<$ Escala $\mathrm{C}<$ Escala $\mathrm{D}<$ Escala $\mathrm{E}<$ Escala $\mathrm{F}$ Escalas cartográficas posibles $\cdots \ldots, \quad \ldots$ Escalas No posibles

Figura 2 Escalas y niveles jerárquiços.

\section{ANÁLISIS DE LA INFORMACIÓN EXISTENTE \\ Para tener una idea general del conocimiento y de la aplicación de los concep- tos de nivel de resolución, clasificación jerárquica, generalización y variables visuales según el nivel de información presentado en el mapa, se óbservó de una serie de mapas con diferentes temáticas y a varias escalas. Además, tanto para la cartografía básica y temática, se hizo un estudio comparativo de mapas de la misma zona a escalas diferentes. La cartografía consultada}


corresponde a la producida en el nivel nacional, tanto por entidades públicas como privadas. Se revisaron aproximadamente más de 300 documentos.

El resultado de esta investigación documental muestra que la presentación de la información se basa preferentemente en clasificaciones de un solo nivel y que se tienen dificultades al establecer los conceptos que entran en juego en una clasificación, como se observa en la figura 3.

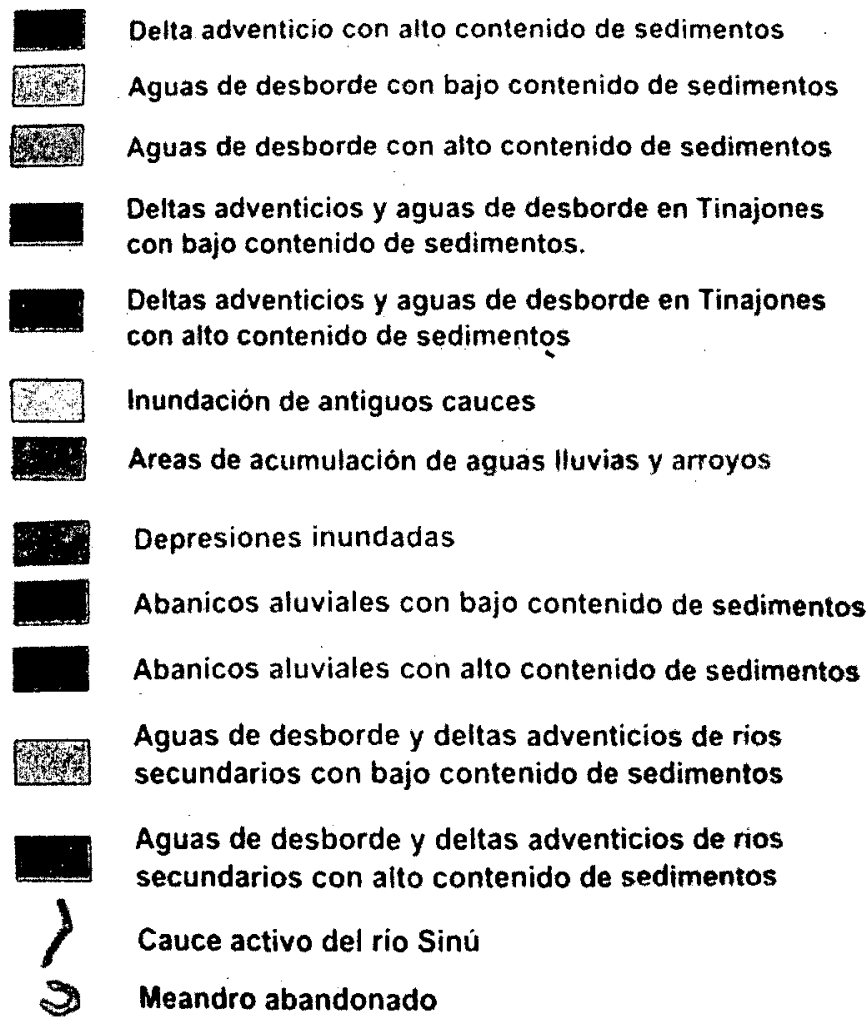

Figura 3 Convenciones del mapa de inundaciones de la cuenca baja del río Sinú.

En esta clasificación plana, en un único nivel o no jerarquizada, se mezclan conceptos de forma (delta), elemento (agua), dinámica (inundación).

Fuente: Instituto Geográfico Agustín Codazzi. Cartografía temática, 1998, p. 160. 
Como se indicó en las figuras 1 y 2, con las clasificaciones en un solo nivel no hay jerarquización y no es posible la generalización conceptual. También se encontró que no hay claridad respecto a que el nivel de resolución de una escala grande debe ser mayor que el nivel de resolución de una escala pequeña (figura 4): dos mapas de escalas muy diferentes presentan la misma resolución. Así mismo, se observó con frecuencia que la aplicación de los principios básicos para el diseño cartográfico es deficiente.

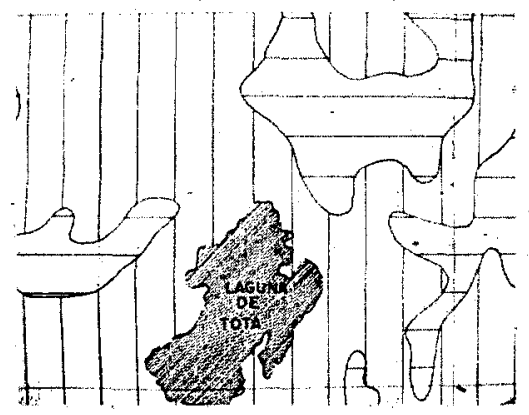

$1: 200.000$

REF
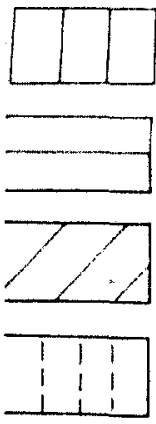

- $\ldots$.

$-\ldots$
Rongo

$0-15$

$15 \cdot 25$

$25 \div 35$

$35-50$

$>50$
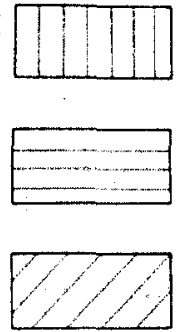

$25-35$

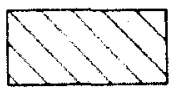

$35-50$

$>$ so

$1: 50.000$

AANGO

$0 \div 15$

$15-25$

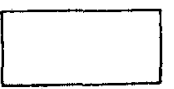

Figura 4 Convenciones de mapas de pendiente a escalas $1: 200.000$ y $1: 50.000$.

Según el concepto de resolución, se esperaría mayor nivel de detalle en el mapa a escala 1:50.000 que en el mapa a escala 1:200.000, es decir, se espera que en un mapa de escala grande tenga mayor número de clases con intervalos 
más estrechos; sin embargo, en ambos mapas el nivel de resolución es igual, lo cual es erróneo.

Fuente: Corporación Autónoma Regional, Boyacá. Mapa de pendientes, 1997; Departamento Administrativo del Medio Ambiente, Mapa de pendientes, 1994.

\section{APLICACIONES}

Con base en los conceptos básicos y de acuerdo con los problemas detectados al analizar materiales cartográficos, y con el apoyo de la bibliografía existente, se propone a continuación cómo enfrentar el proceso de generalización cartográfica. La propuesta procedimental para la generalización conceptual y estructural en cartografía básica y temática, que facilita aplicar correctamente los conceptos mencionados, se basa en la clasificación jerárquica y los conceptos de nivel de resolución y escala, y se presenta en el mapa conceptual de la figura 5.

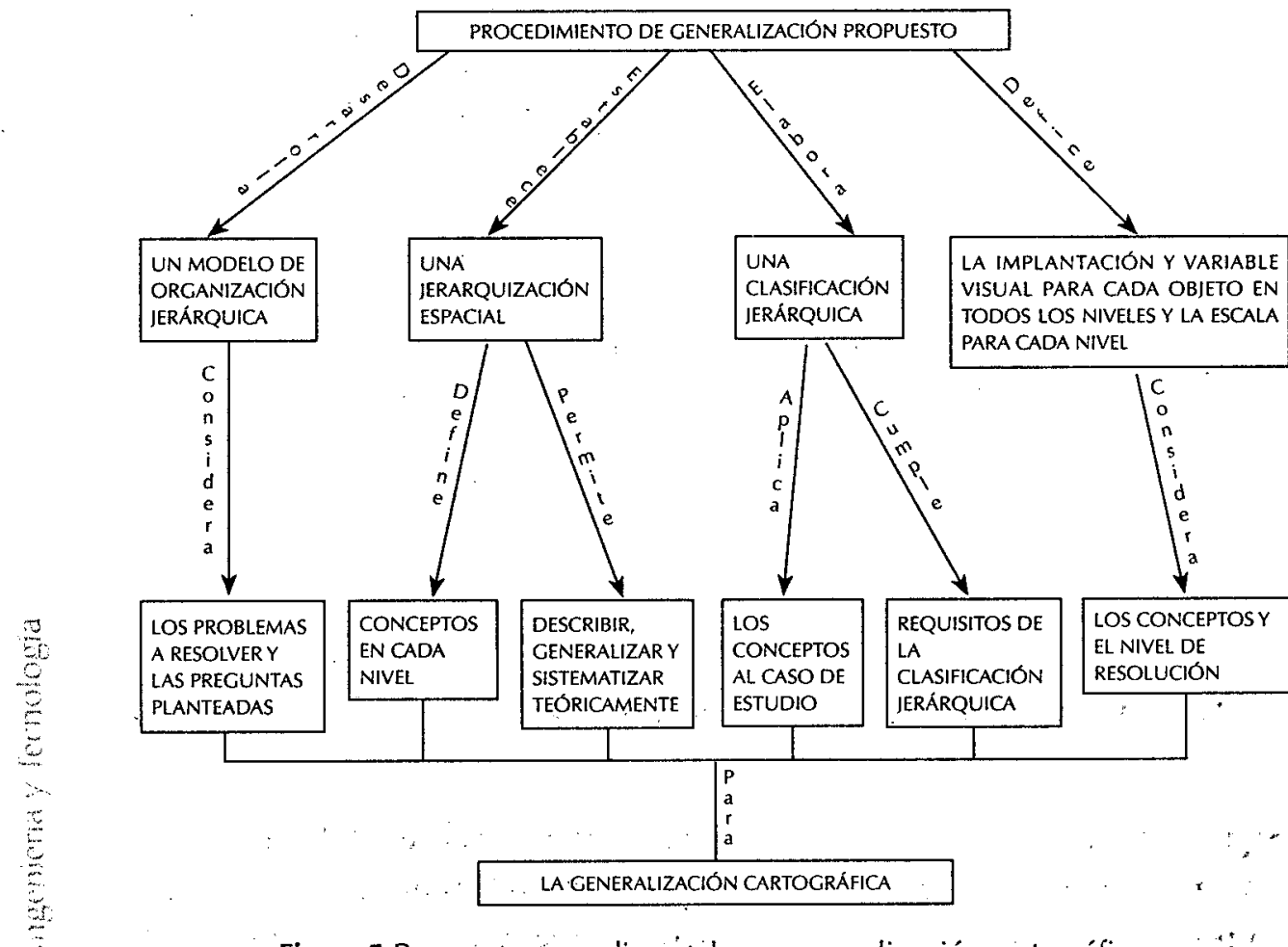

Figura 5 Propuesta procedimental para generalización cartográfica. 
A continuación, se presenta una serie de aplicaciones de generalización en cartografía temática en las que se desarrolla el procedimiento propuesto. Estos ejemplos'de aplicación tienen como característica fundamental la organización jerárquica, puesto que es el requisito para establecer claridad respecto al nivel de resolución y la escala del mapa. Por tanto, el énfasis de la generalización el punto de vista conceptual, y los ejercicios presentan diferentes niveles conceptuales para cada escala.

\section{Generalización cartográfica de una red de drenaje}

El ejemplo de aplicación para generalización cartográfica es tomado de Torres y Join (1987), quien elabơró el Diagnóstico geográfico para una cuenca de la altillanura del Vichada. Esta red de drenaje está jerarquizada de acuerdo con la taxonomía planteada por Horton. El esquema de órdenes de corrientes fue desarrollado a principios de la década de 1940 por el ingeniero hidráulico e hidrólogo estadounidense Robert Horton. Esta taxonomía de las corrientes establece una estructura jerárquica, como se muestra en la figura 6.

Una corriente que constituye la cabecera de un río y carece de afluentes pertenece a la primera categoría. Dos corrientes de primera categoría se unen para formar una corriente de segunda categoría; dos corrientes de segunda categoría se unen para formar una de tercera categoría, y àsí sucesivamente. Este sistema de clasificación muestra cómo se une cada corriente a la red y cómo está conectada la red en su conjunto.

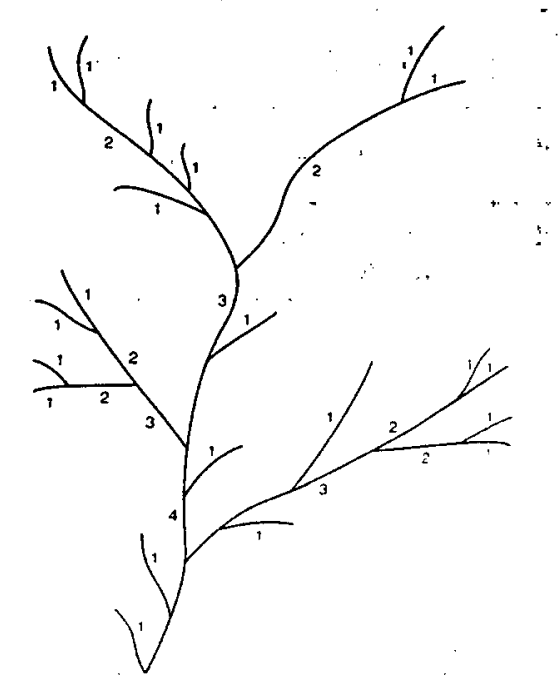

Figura 6 Jerarquización de drenajes. 
La red de drenaje de esta cuenca corresponde a una jerarquía que contiene siete niveles relacionados con el orden o categoría del drenaje. La forma de implantación es lineal y la variable visual que se debe emplear es valor.

En este ejemplo, la generalización conceptual consiste en ir eliminando el último nivel jerárquico y asignar una escala menor, como se muestra en el cuadro 1. La generalización estructural consiste en presentar a escalas menores líneas más simplificadas.

En la figura 7 se observa parte de los primeros cuatro rangos de escala mayores; en la figura 8, parte de los últimos cinco rangos.

Cuadro 1 Máximo nivel de jerarquía de drenaje incluido según la escala.

\begin{tabular}{|c|c|c|}
\hline Rango de escalas & $\begin{array}{c}\text { Niveles } \\
\text { jerárquicos }\end{array}$ & $\begin{array}{c}\text { Ordenes } \\
\text { de drenaje }\end{array}$ \\
\hline $\begin{array}{c}1: 25.000>1: E \\
1: 100.000\end{array}$ & $\begin{array}{c}7 \text { niveles (máx. } \\
\text { resolución) }\end{array}$ & $\begin{array}{c}\text { Ordenes } 1,2,3, \\
4,5,6 y 7\end{array}$ \\
\hline $\begin{array}{c}1: 100.000>1: E \\
1: 250.000\end{array}$ & 6 niveles & $\begin{array}{c}\text { Ordenes } 2,3,4, \\
5,6 y 7\end{array}$ \\
\hline $\begin{array}{c}1: 250.000>1: E \\
1: 400.000\end{array}$ & 5 niveles & $\begin{array}{c}\text { Órdenes } 3,4,5, \\
6 y 7\end{array}$ \\
\hline $\begin{array}{c}1: 400.000>1: E \\
1: 700.000\end{array}$ & 4 niveles & $\begin{array}{c}\text { Ordenes } 4,5,6 \\
y\end{array}$ \\
\hline $\begin{array}{c}1: 700.000>1: E \\
800.000\end{array}$ & 3 niveles & Ordenes 5,6 y 7 \\
\hline $\begin{array}{c}1: 800.000>1: E \\
1: 900.000\end{array}$ & 2 niveles & Ordenes 6 y 7 \\
\hline $\begin{array}{c}1: 900.000>1: E \\
1: 1000.000\end{array}$ & 1 nivel (mín. & resolución)
\end{tabular}



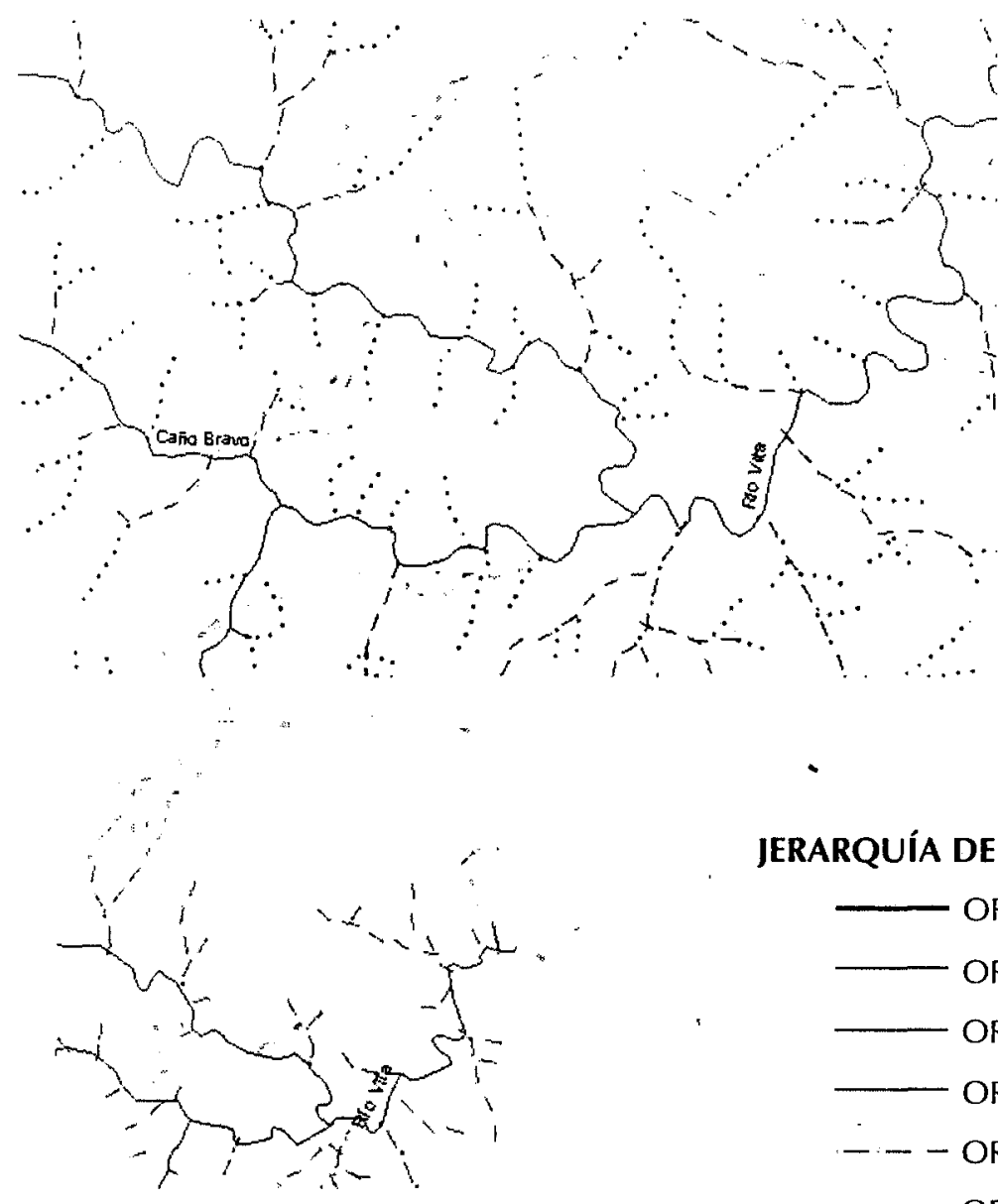

JERARQUÍA DE DRENAJES
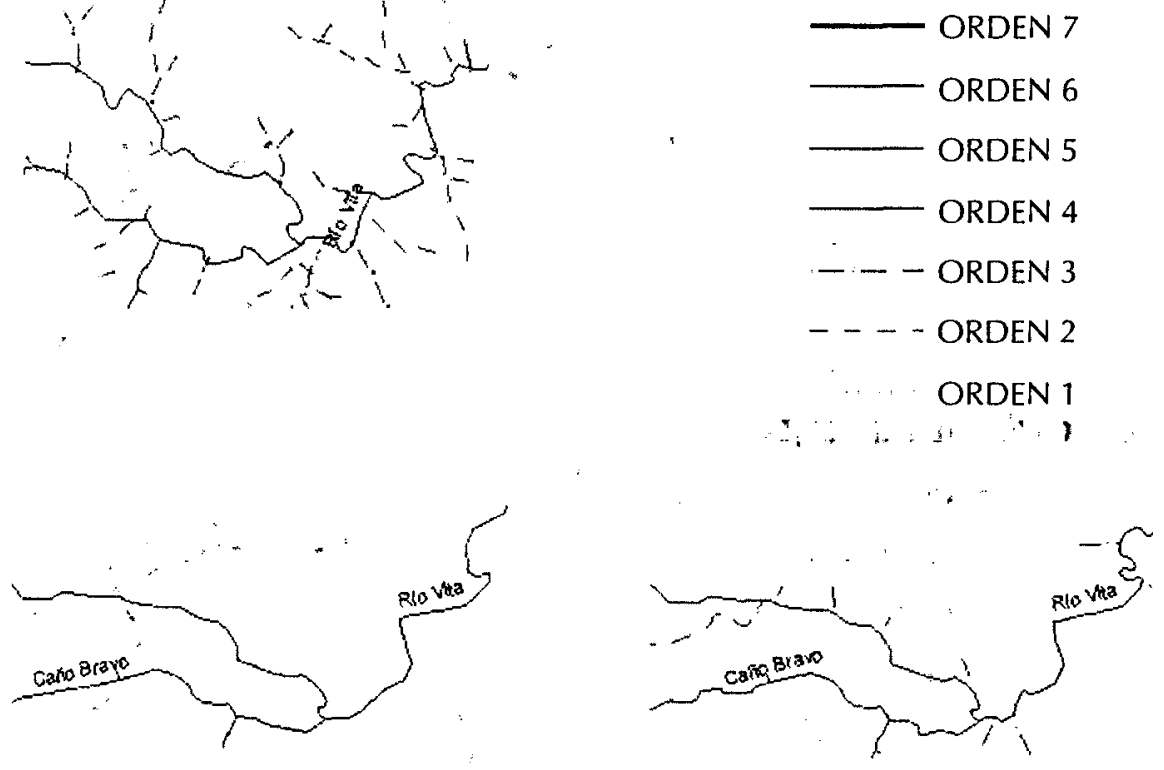

Figura 7 Generalización estructural y conceptual de los drenajes. 


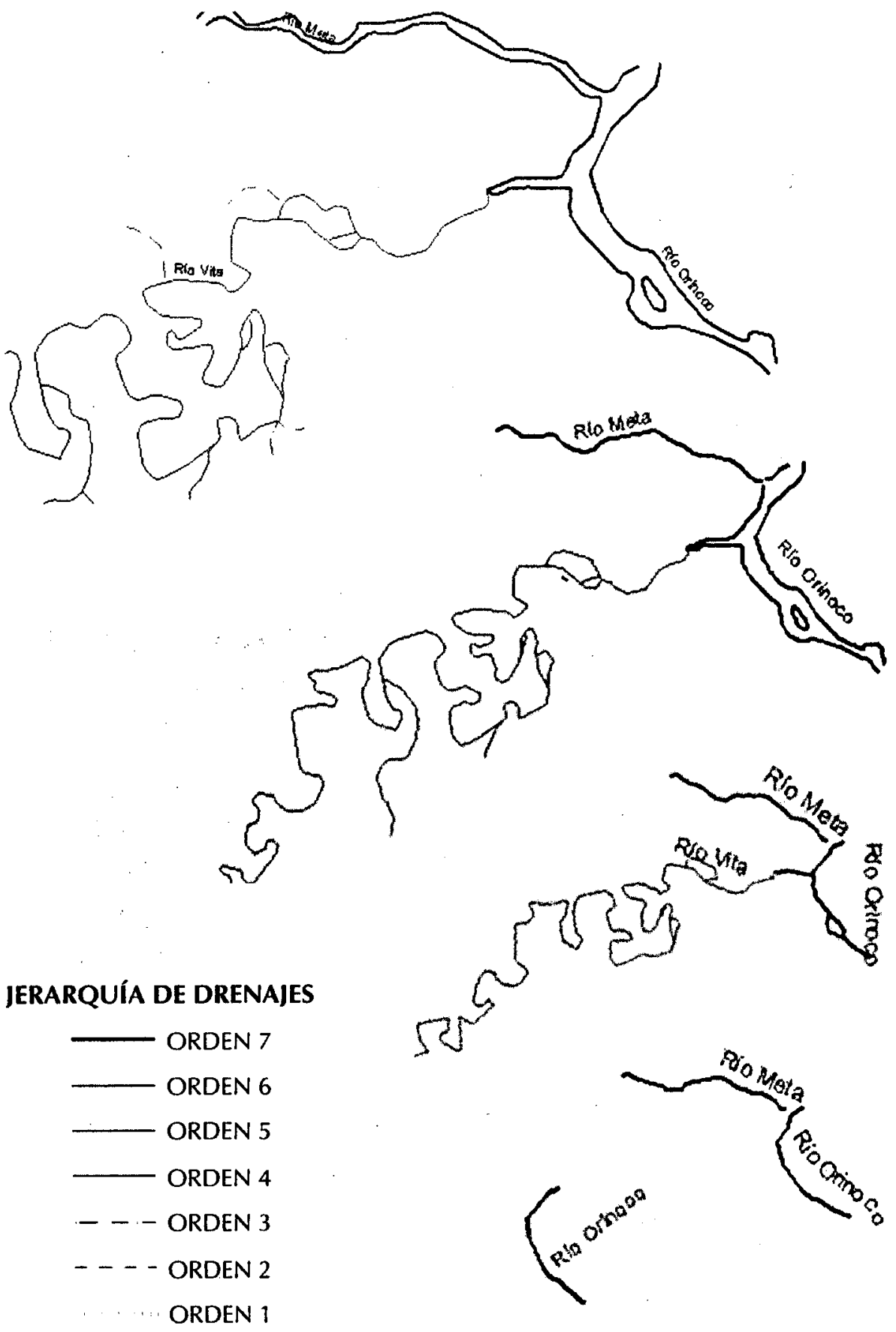

Figura 8 Generalización estructural y conceptual de los drenajes: 
Generalización cartográfica de la división político-administrativa de Colombia

La división político-administrativa de Colombia es un ejemplo típico de estructura jerárquica. En la actualidad, para la administración del Estado y la representación política, Colombia está dividida en 32 departamentos y 1.092 municipios. Los departamentos son entidades territoriales descentralizadas que tienen funciones administrativas y cumplen un papel de intermediación entre la nación y los municipios, tienen autonomía administrativa, personería jurídica y patrimonio autónomo; los municipios también son entidades territoriales descentralizadas que tienen funciones administrativas, autonomía administrativa, personería jurídica y patrimonio autónomo. El municipio es considerado el núcleo fundamental de la organización político-administrativa. Además, se presenta el caso en el que en un mismo departamento los municipios se agrupan en provincias. Por ejemplo, para el caso específico del Departamento de Cundinamarca, la definición provincial establecida en la Ordenanza 023 de 1998, señala que en este existen 15 provincias y 116 municipios. Es importante señalar que lós municipios están conformados por veredas, como lo muestra el esquema de la clasificación jerárquica de la figura 9.

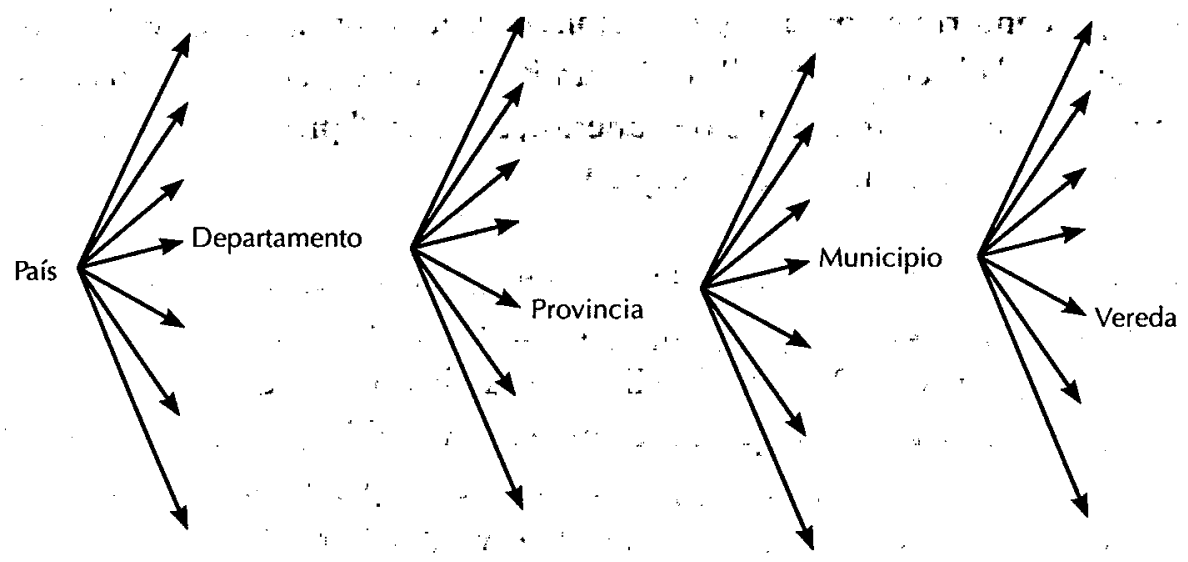

Figura 9 Jérarquía político-administrativa nacional.

El nivel correspondiente a la provincia se tomó tal cual aparece en la referencia citada. 
Este ejemplo cuenta como máximo nivel de resolución la división municipal; es decir, no incluye veredas. La generalización conceptual consiste en que.a partir de los municipios se generaliza y se conforman los holons de nivel superior denominados provincias; éstas se generalizan y dan origen a los holons de nivel superior denominados departamentos; estos se generalizan y constituyen el holon de nivel superior denominado país. En el cuadro 2 se observan los niveles jerárquicos que se representan en los rangos de escala.

Cuadro 2 Nivel jerárquico de división político administrativa según la escalá.

\begin{tabular}{|c|c|}
\hline Nivel jerárquico & Rango de escala \\
\hline Municipio & $1: 25.000>1: \mathrm{E} \geq 1: 800.000$ \\
\hline Provincia & $1: 800.000>1: \mathrm{E} \geq 1: 3.000 .000$ \\
\hline Departamento & $1: 3.000 .000>1: \mathrm{E} \geq 1: 12.000 .000$ \\
\hline Colombia & $1: 12.000 .000>1: \mathrm{E} \geq 1: 100.000 .000$ \\
\hline
\end{tabular}

La generalización estructural consiste en simplificar los bordes de los polígonos que conforman los diferentes holons en la jerarquía. Esta generalización se aprecia al observar el detalle de los límites: el mayor se presenta en el nivel municipal; el menor, en el holon superior, es decir, al presentar solamente el polígono del país a una escala pequeña.

Por el tipo de información de esta jerarquía, no se requiere emplear variable visual para diferenciar, por ejemplo, un municipio de otro, sino que basta con los topónimos. Para la visualización de los mapas que presentan los diferentes conceptos jerárquicos se emplea la variable valor. En la figura 10 se presentan sectores de los mapas en los que se aprecian los resultados de los procesos de generalización conceptual y estructural. La generalización conceptual ocurre al presentar en cada mapa un concepto cada vez de mayor jerarquía, es decir, de municipios a provincias, de provincias a departamentos, y de departamentos al país. La generalización estructural se aprecia en que los límites de las zonas son cada vez más simples. 

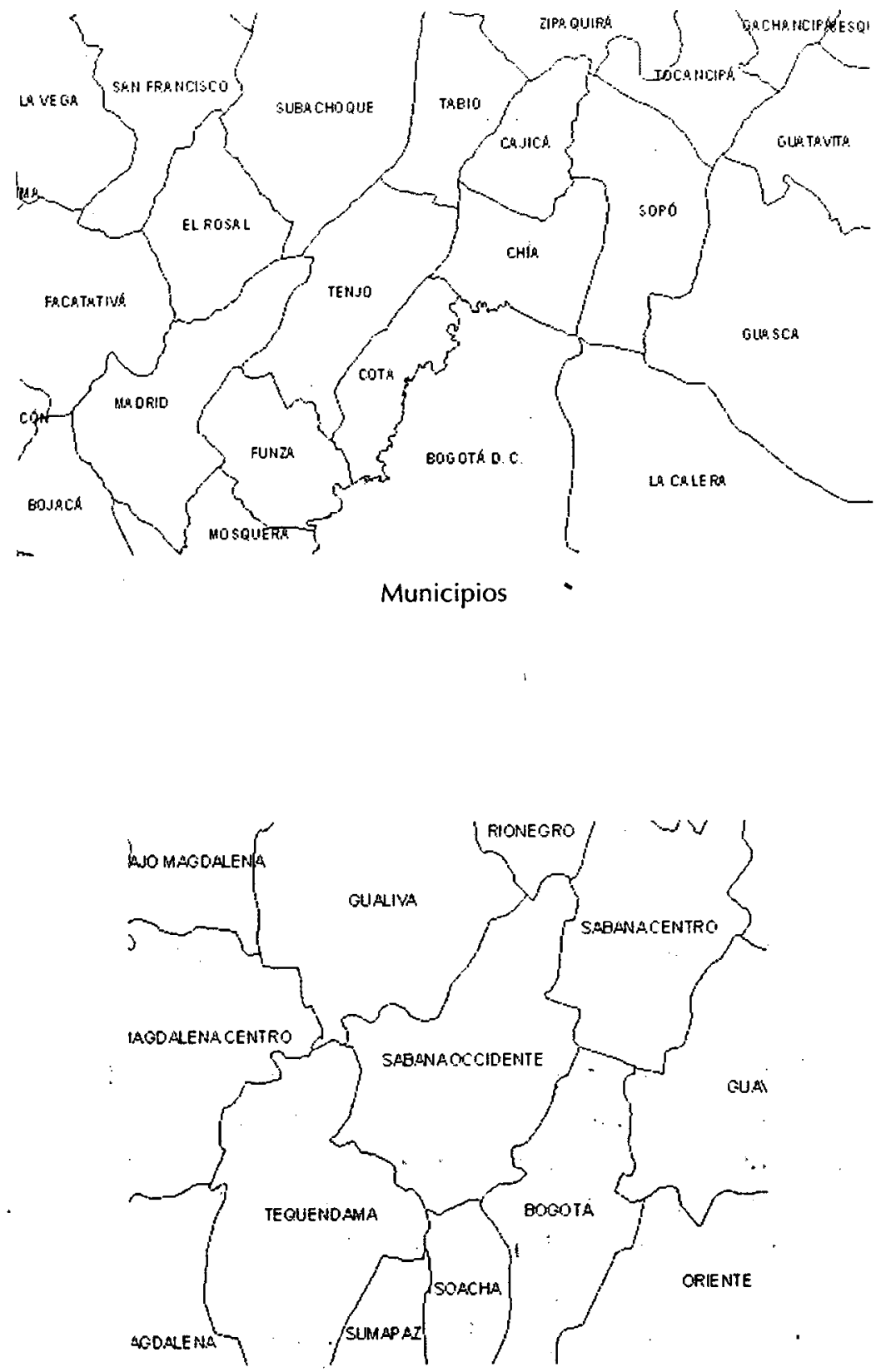

Provincias 

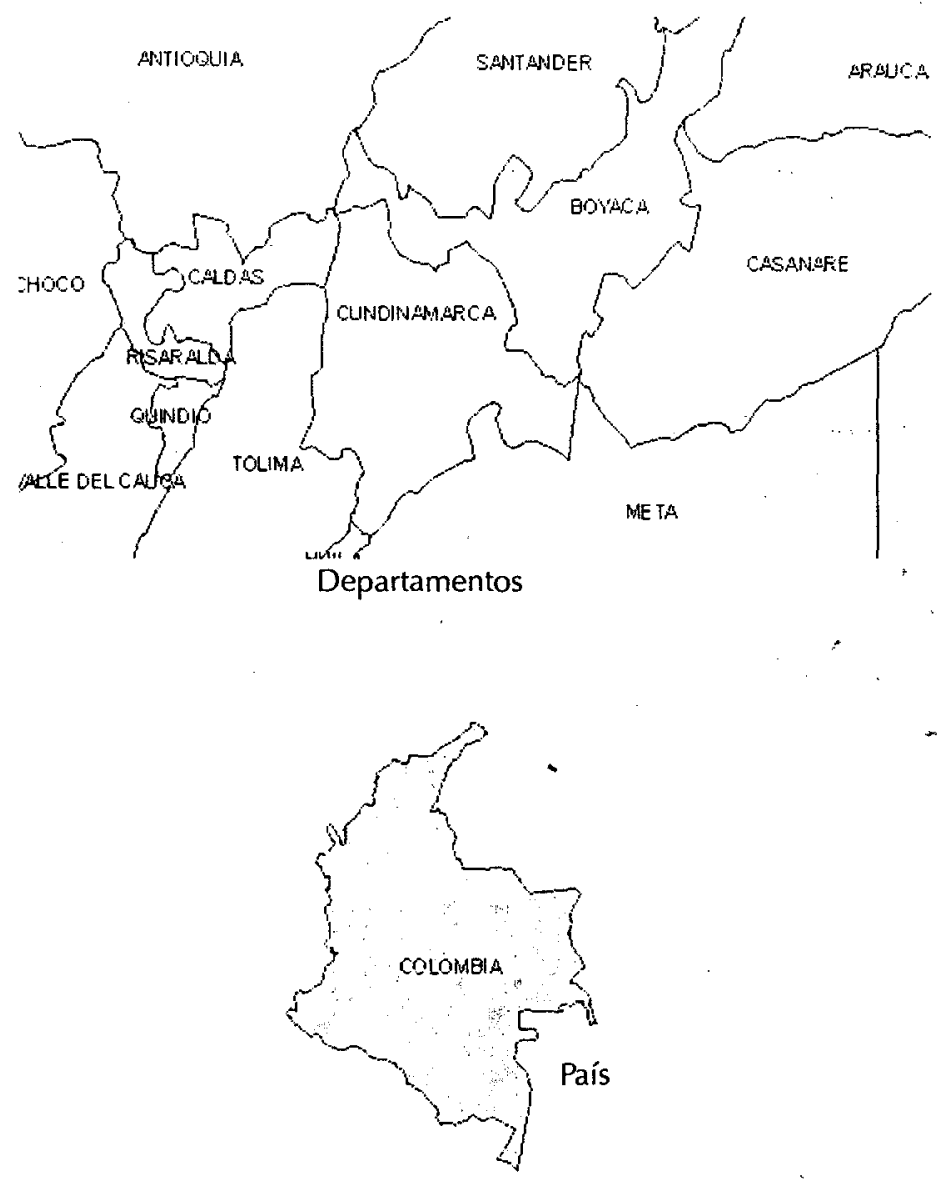

Figura 10 Generalización de la división político-administrativa.

\section{Generalización cartográfica de la jerarquía urbana en Colombia}

La idea de una jerarquía del espacio urbano que resulta del crecimiento de ciudades y el desarrollo de sistemas de ciudades es un principio lógico de orden general. La organización jerárquica es básica para describir y medir la importancia de las funciones urbanas a lo largo de muchas escalas espaciales. El ejemplo clásico en la ciudad está relacionado con funciones rutinarias como la venta al por menor y los servicios comerciales, cuya frecuencia y escalas de provisión están ligadas a las mismas características de los lugares donde se llevan a cabo. La misma estructura jerárquica existe para el sistema educativo, y otros sistemas, los cuales están diferenciados por el nivel de detalle en sus funciones. 
Este ejemplo de generalización cartográfica toma como base la jerarquía de la red nacional urbana, realizada por la Subdirección de Geografía del Instituto Geográfico Agustín Codazzi (Londoño et al., 1986). En el documento se explica que la influéncia que ejerce ún centro èn el interior de una región depende de su dinamismo y de su podér de atracción, en los que la red de comunicaciones y las condiciones naturales del medio juegan un papel importante. Se utilizaron doś metodologías para establecer la jerarquía de los centros urbanos nacionales: el método dé índices de participación y el método de Guttman.

La jerarquización por el método de índices de participación tiene en cuenta la variedad, calidad y cantidad de lós serviciós de cada centro urbano, y la posibilidad de atender lós réquérimién'toś de su clientela, tanto urbana cómo la que vive en su área de influencia (Londoño et al., 1986:147). La jerarquización por el método de centralidad dé Guttman tiené en cúenta la cantidad y calidad de equipamientos urbanos de tipo administrativo, público, comercial y bancario, social, cultural y medios masivos de comunicación (Londoño et al., 1986:162). Analizando los resultados de las jerarquías obtenidas, se determinaron los siguientes niveles jerárquicos: 1.Metrópoli nacional (MN), 2. Metrópoli regional (MR), 3. Centro subregional especial (CSRE), 4. Centro subregional (CSR), 5. Centro de relevo principal (CRP), 6. Centro de relevo (CR), 7. Centro local principal (CLP) y 8. Centro local (CL).

Un esquema de la jerarquía urbana, que muestra los primeros seis niveles, se presenta de manera sencilla en la figura 11. Es importante aclarar que la jerarquía no excluye que existan relaciones entre holons que no se vean conectados, es decir, por ejemplo, que no exista relación alguna entre Popayán y Villavicencio.

Para este ejemplo de aplicación, los centros urbanos se representan con implantación puntual, y la variable visual empleada es valor. La generalización conceptual consiste en ir eliminando el último nivel de la jerarquía a partir del máximo nivel de resolución, que incluye todos lo niveles jerárquicos, hasta llegar a representar sólo el primer nivel. La escala de representación para cada nivel corresponde con el nivel de resolución que se ofrece en cada mapa, como se presenta en el cuadro 3. 


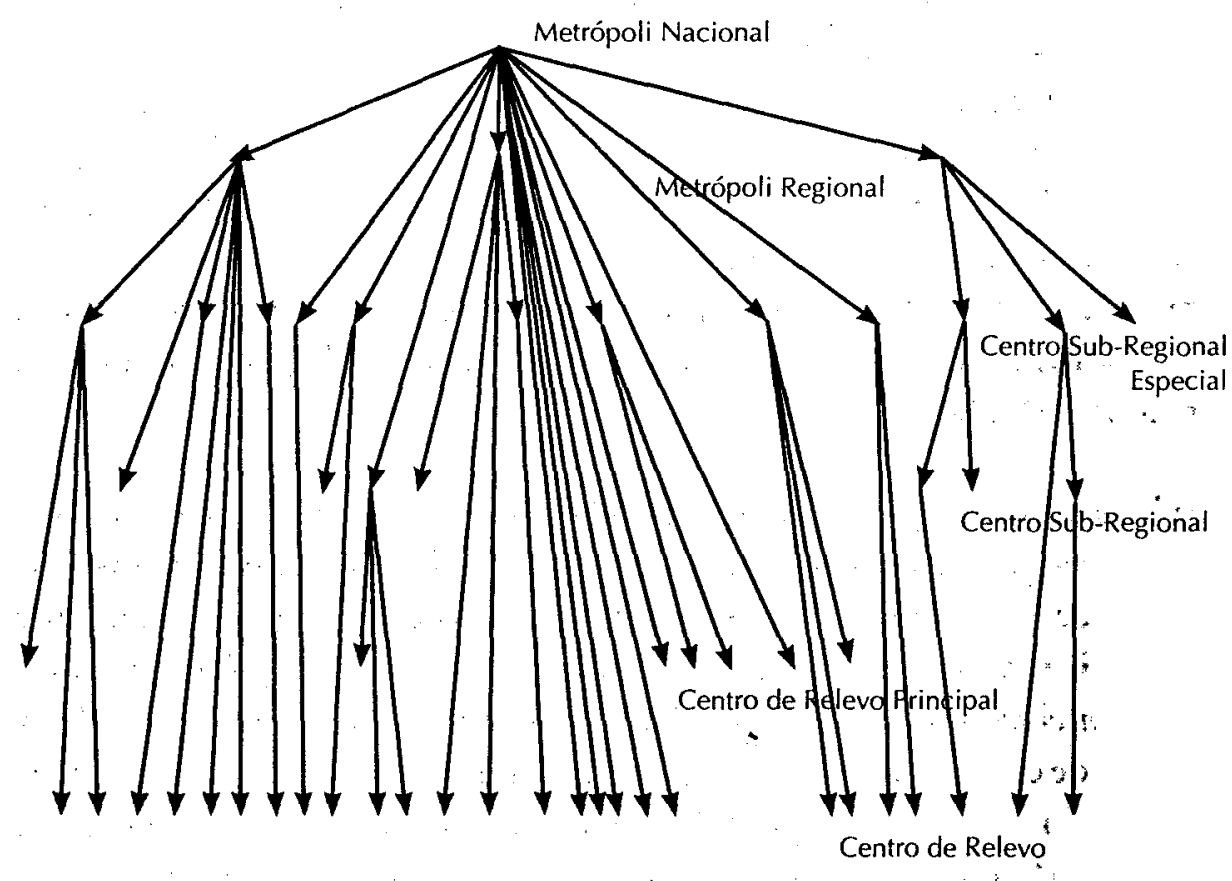

Figura 11 Esquema jerárquico de centros urbanos (premapa).

Cuadro 3 Niveles jerárquicos incluidos en cada rango de escalas.

\begin{tabular}{|l|l|}
\hline \multicolumn{1}{|c|}{ Escala } & \multicolumn{1}{|c|}{ Niveles jerárquicos incluidos } \\
\hline $1: 200.000>1: E \geq 1: 1: 500.000$ & $\mathrm{CL}, \mathrm{CLP}, \mathrm{CR}, \mathrm{CRP}, \mathrm{CSR}, \mathrm{CSRE}, \mathrm{MR}, \mathrm{MN}$ \\
\hline $1: 1.500 .000>1: \mathrm{E} \geq 1: 2.500 .000$ & $\mathrm{CLP}, \mathrm{CR}, \mathrm{CRP}, \mathrm{CSR}, \mathrm{CSRE}, \mathrm{MR}, \mathrm{MN}$ \\
\hline $1: 2.500 .000>1: \mathrm{E} \geq 1: 4.000 .000$ & $\mathrm{CR}, \mathrm{CRP}, \mathrm{CSR}, \mathrm{CSRE}, \mathrm{MR}, \mathrm{MN}$ \\
\hline $1: 4.000 .000>1: \mathrm{E} \geq 1: 7.000 .000$ & $\mathrm{CRP}, \mathrm{CSR}, \mathrm{CSRE}, \mathrm{MR}, \mathrm{MN}$ \\
\hline $1: 7.000 .000>1: \mathrm{E} \geq 1: 12.000 .000$ & $\mathrm{CSR}, \mathrm{CSR}, \mathrm{CSRE}, \mathrm{MR}, \mathrm{MN}$ \\
\hline $1: 12.000 .000>1: \mathrm{E} \geq 1: 25.000 .000$ & $\mathrm{CSRE}, \mathrm{MR}, \mathrm{MN}$ \\
\hline $1: 25.000 .000>1: \mathrm{E} \geq 1: 35.000 .000$ & $\mathrm{MR}, \mathrm{MN}$ \\
\hline $1: 35.000 .000>1: \mathrm{E} \geq 1: 100.000 .000$ & $\mathrm{MN}$ \\
\hline
\end{tabular}


Este ejemplo de aplicación no requiere generalización estructural, pero se presenta sobre las zonas del ejemplo de división político-administrativa, las cuales se generalizaron tanto conceptual como estructuralmente. En la figura 12 se presentan partes de dos mapas y un mapa: una parte corresponde al máximo nivel de resolución (los municipios); la otra pertenece al mapa que contiene cinco niveles jerárquicos (los departamentos). A la derecha, se presenta el mapa con tres niveles jerárquicos.
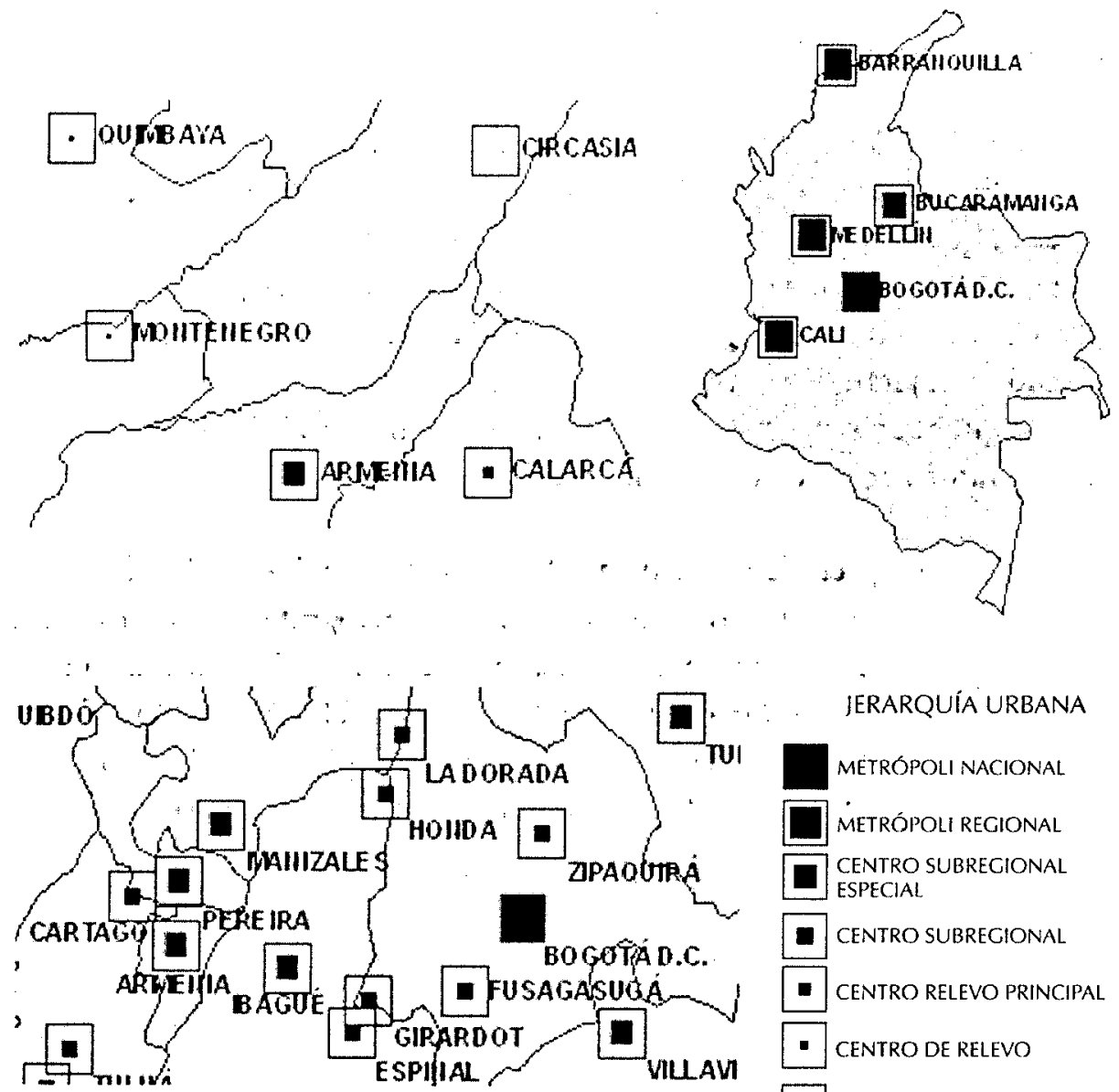

JERARQUÍA URBANA METRÓPOLI NACIONAL METRÓPOLI REGIONAL CENTRO SUBRECIONAL ESPECIAL

- Centro subrecional

- CENTRO RELEVO PRINCIPAL

- Centro de Relevo

$\square$ CENTRO LOCAL PRINCIPAL
CENTRO lOCAL

Figura 12 Generalización de la jerarquía urbana. 


\section{Generalización cartográfica de las formas de ocupación del espacio}

Este ejemplo de aplicación es tomado de un ejercicio de generalización para las formas de ocupación del espacio, desarrollado teóricamente (A. Flórez, 2004) en la asignatura de Cartografía de la Maestría en Geografía del EPG. Para una zona de estudio supuesta, se presenta una serie de formas de ocupación del espacio que se clasifican jerárquicamente. Esta taxonomía de formas de ocupación del espacio contiene cinco niveles, para cada uno de los cuales se establece una escala de representación.

La generalización cartográfica ocurrirá a medida que se vaya suprimiendo el nivel inferior; la generalización conceptual implica eliminar cada vez un nivel de información, y la generalización estructural implica simplificar las líneas. Para representar todos los niveles taxonómicos se requiere una escala grande; a medida que se va eliminando de la representación cartográfica el último nivel jerárquico, la escala del mapa disminuye. La escala más grande será la que represente hasta el quinto nivel, y no se podrá representar esta misma información en escalas mayores.

Una vez se tenga claridad de los niveles jerárquicos y las escalas de representación, se determina cómo debe resolverse cartográficamente, es decir, se aplican las variables visuales correctas: para el nivel de información diferencial se emplean color, forma y orientación; para el nivel de información ordenado se emplea valor, como se esquematiza en la figura 13. En las figuras 14 y 15 se muestran partes de la generalización de este ejemplo. 


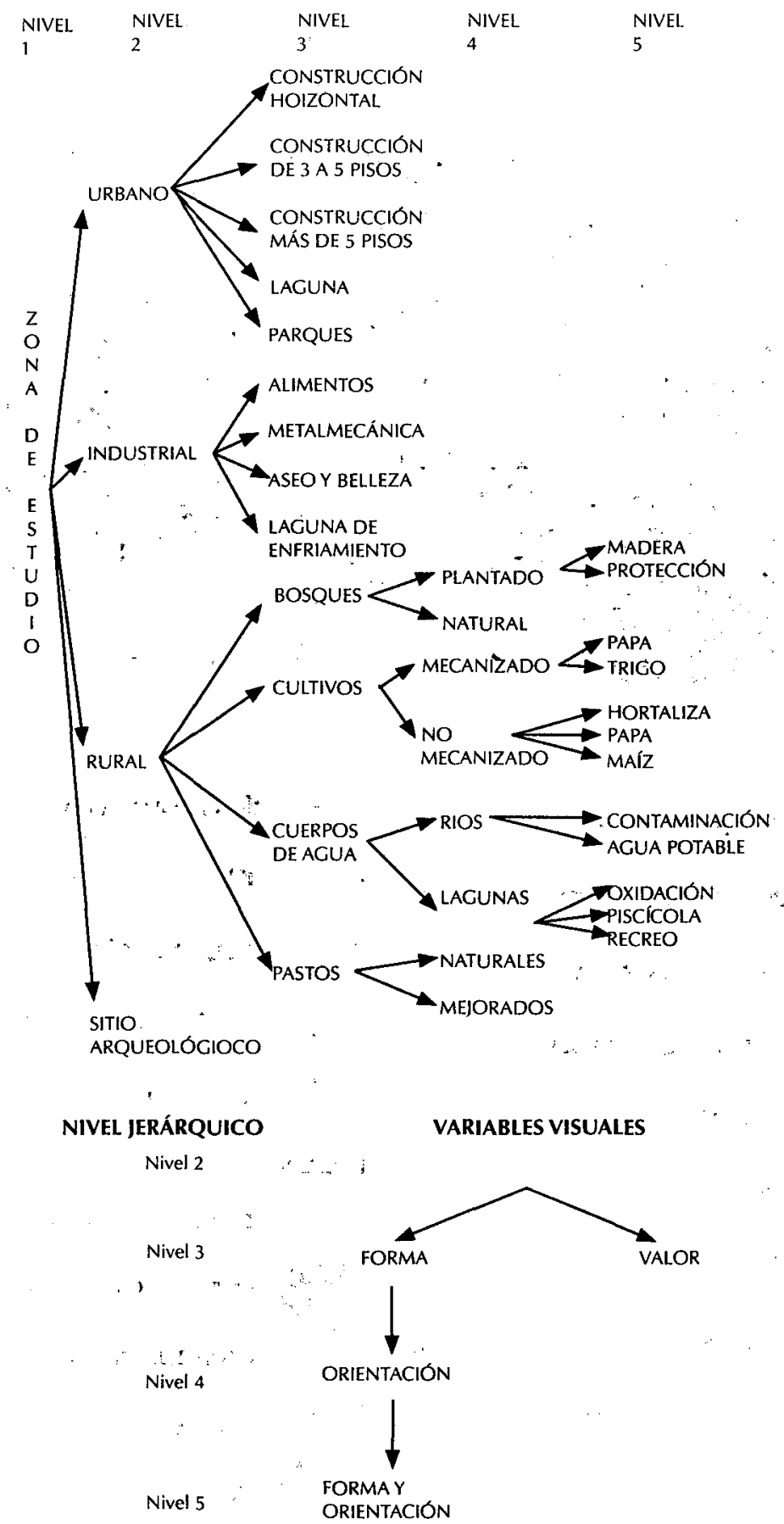

Figura 13 Clasificación jerárquica y variables visuales para formas de ocupación del espacio. 


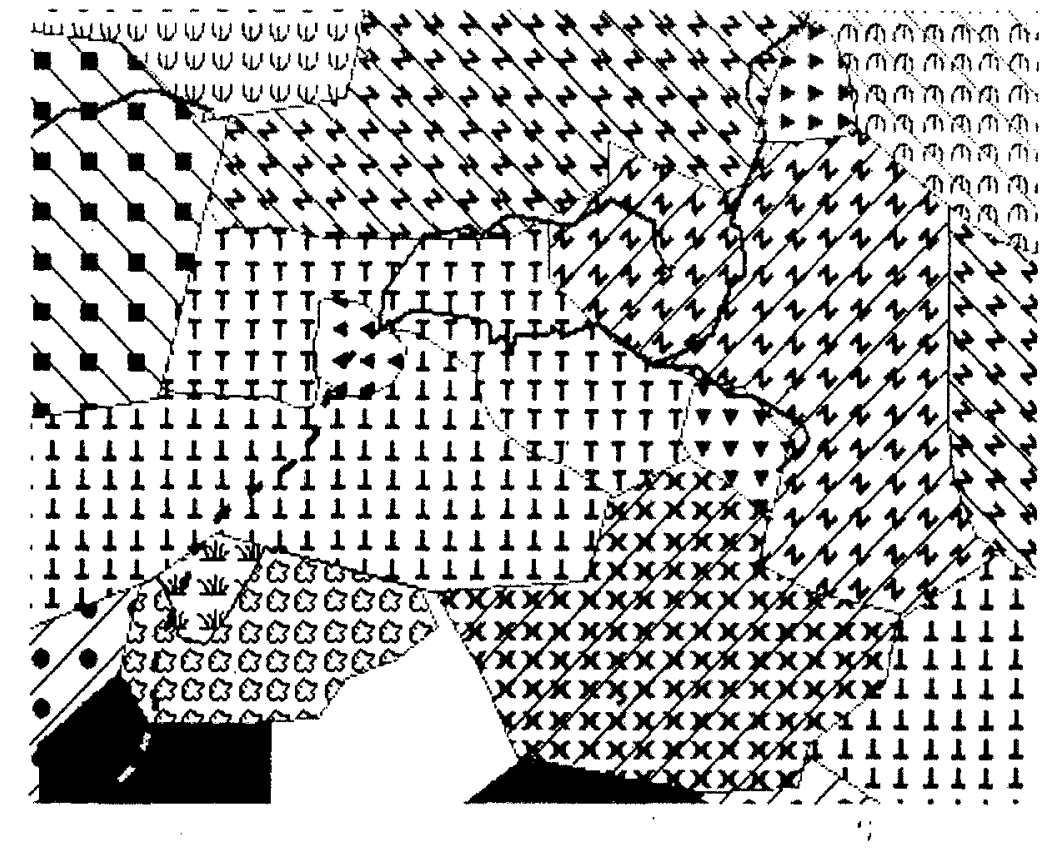

RURAL

NWWy BOSQUE NATURAL

GMMOSQ BLANTADO MADERA

haming

PAPA MECANIZADA

STRIGO MECANIZADO

GORTALIzAS.

Exixy MAIZ

PaPA

Fit PASTOS MEJORADOS

211

FT: PASTOS NATURALES

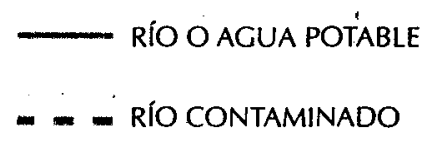

ed laguna piscícola

LaGuna de OXIDACIÓN

144 LAGUNA DE RECREO

URBANO

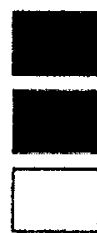

CONSTRUCCIÓN DE 3 A 5 PISOS

CONSTRUCCIÓN DE MÁS DE 5 PISOS

CONSTRUCCIÓN HORIZONTAL

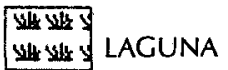

QOQ PARQUE

Figura 14 Generalización de formas de ocupación del espaciiò. 


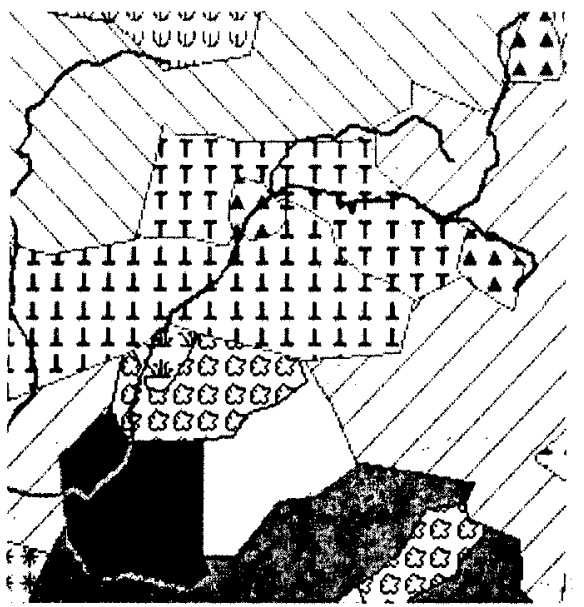

RURAI.

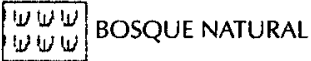

CULTIVO MECANIZADO

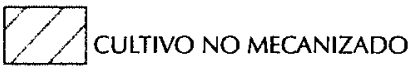

A. A LAGUNAS

$\left[\begin{array}{lll}1 & 1 & 1 \\ 1 & 1 & 1\end{array}\right]$ PASTOS MEJORADOS

TT T T PASTOS NATURALES

TTT

RÍOS
URBANO

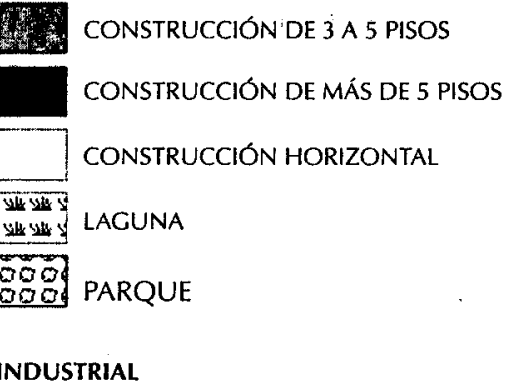

ais

(1) 


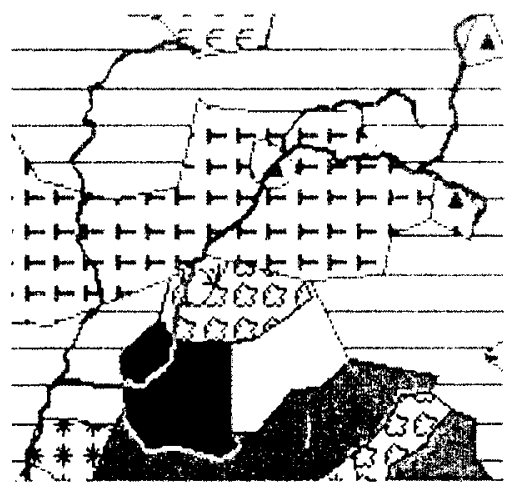

RURAL

A.A CuERPOS DE ACUA

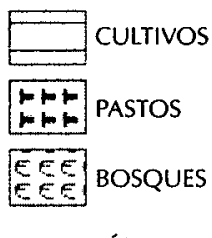

RÍOS
URBANO

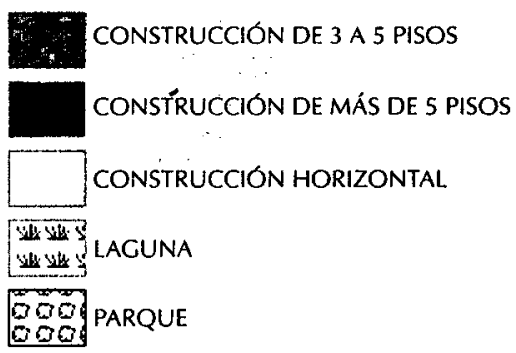

\section{INDUSTRIAL}

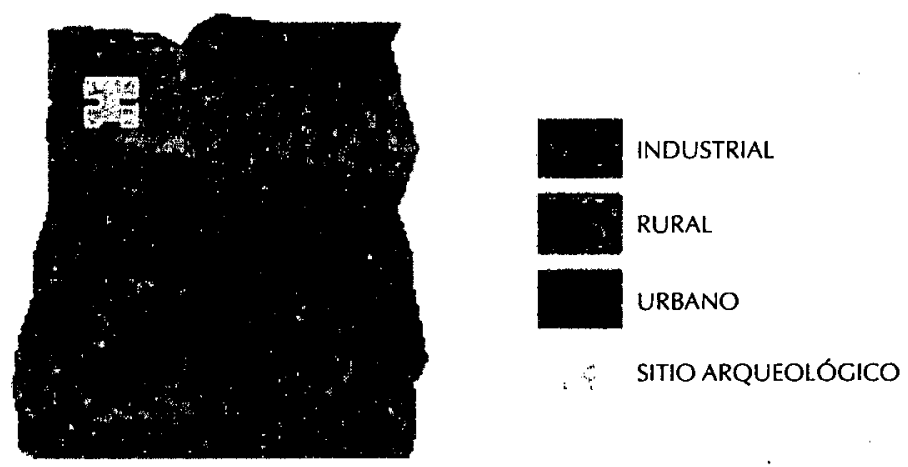

Figura 15 Generalización de formas de ocupación del espacio. 


\section{CONCLUSIONES}

Cuando se reflexiona sobre la interacción del mundo humano de la experiencia con el mundo físico de la existencia, es inevitable considerar la organización espacial. La comprensión de los fenómenos, sistemas y procesos es esencial para entender la organización espacial a fin de orientar la intervención de los grupos sociales. Pensar y conceptualizar sobre el espacio es una de las principales tareas del geógrafo, que se vale de herramientas como la cartografía, lá cual facilita la expresión del espacio que requiere ser explicado conceptualmente, permite reflexionar acerca de la relación de las estructuras y formas espaciales, presenta el pensamiento del pasado y del presente, y colabora en la construcción del pensamiento futuro, y facilita la reproducción de la cultura que lleva dentro.

En la conceptualización del espacio geográfico existen diversos niveles; al cambiar el nivel conceptual cambia el contenido, expresado por el nivel de resolución que puede ir de lo detallado a lo general. Para presentar los diferentes niveles de resolución es necesario recurrir a diversas escalas, por lo cual es importante saber cuáles son los nuevios contenidos en cada nivel de resolución y tener presente que al cambio de escala le corresponde un cambio en el nivel de concepción, es decir, la escala se asocia a un espacio de concepción establecido previamente.

El espacio ha sido y será tema de eśtudio én la geografía, al igual que la discusión de los diferentes niveles de resolución que se manifiestan en espacios más o menos detallados. En esta propuesta de generalización cartográfica y los ejemplos de aplicación desarrollados, se evidencia la importancia de establecer una jerarquización espacial para definir los conceptos que orientan el nivel de resolución y permiten varios y específicos niveles de observación espacial.

El espacio geográfico se puede explicar como una serie de "sistemas encajados" unos en otros, dando la posibilidad de seleccionar espacios más o menos complejos. Con la organización jerárquica se establece la dependencia funcional y la pertenencia espacial, es decir, se especifican las funciones que ejercen unos conceptos sobre otros y las relaciones de doble sentido, y se reconoce que hay un espacio que abarca todo y que cada uno tiene su espacio. Con la 
aplicación de las nociones de clasificación jerárquica, nivel de resolución y escala en la propuesta de generalización cartográfica, se tiene la posibilidad de asignar a cada nivel jerárquico la dimensión espacial suficiente para expresar el concepto específico.

Para un manejo correcto de las escalas cartográficas es necesario tener presente que unas escalas determinadas exigen unos conceptos de cierta resolución. Esto no se puede confundir con la preocupación que invade a muchos profesionales al hacer un mapa respecto al tamaño mínimo de los elementos que se van a representar, aunque éste es un problema de équilibrio gráfico del mapa. El problema prioritario por resolver en el manejo de cartografía es cuál es el concepto de mayor resolución dentro de la jerarquía que se va a representar, es decir, conceptualmente qué es lo más pequeño que se muestra en el mapa.

Con base en la investigación documental de materiales cartográficos producidos por diversas entidades nacionales (IGAC, DANE, Ingeominas, CAR, DAMA.), se encontró que la presentación de la información se basa preferentemente en clasificaciones horizontales o planas y en muy pocos casos consideran clasificaciones con más de un nivel. Además, se halló que existen dificultadades al establecer los conceptos que entran en juego en una clasificación.

De manera más o menos generalizada, se percibe la falta de aplicaciones conceptuales y metodológicas a los diferentes objetos temáticos de estudio que permitan establecer niveles jerárquicos a partir de diferentes nivelés de resolución en función de la información, y específicamente "niveles de resolución espacial". También se advierte la falta de conocimiento de las variables visuales que permiten diseñar la información cartográfica de acuerdo con los niveles de la información y las formas de implantación cartográfica.

Con base en las deficiencias señaladas, se planteó la necesidad de organizar la información a partir de conceptos como "sistemas encajados", o "sistemas complejos", "teoría jerárquica”, jerarquía de holons u "holarquía”, de manera que, teniendo claridad respecto a los niveles de resolución, se establezca una organización jerárquica para generalizar cartográficamente, siguiendo el procedimiento propuesto. 
Este proceso académico de investigación se materializa en la propuesta conceptual y procedimental de aplicaciones para el manejo de cartografía. La aplicación está en cómo manejar la información cartográfica para lograr un proceso de generalización cartográfica desde una conceptualización geográfica.

Definida la base conceptual anterior, se aplicó a ejemplos de cartografía temática: generalización de la división político-administrativa de Colombia, generalización de la jerarquía urbana; generalización de una red de drenaje, generalización de geosistemas, generalización de formas de ocupación del espacio y generalización de la división administrativa en Bogotá, en los que se muestra la clasificación jerárquica, los contenidos en fünción de la escala cartográfica, las variables visuales referidas a los niveles jerárquicós y los mapas generalizados.

Uno de los resultados de la çlasificación jerárquica es la leyenda de los mapas en distintos nivéles escalarès. Esto debe éstár unido a un empleo correcto de variables visuales para, que adémás de reflejar el nivèl de là información, reflejen fielmente la noción "de clasificación jerárquiça en los espacios más o menos détallados.

Con las clasificaciones de un solo nivel, las más empleadas; no es posible jerarquizar; por tanto, no es posible la generalización conceptual. Con lo anterior no se indica que al elaborar cartografía sea necesario generalizar, aunque sí es una buena práctica científica clasificar jerárquicamente los objetos de estudio cuando esto sea posible o necesario de acuerdo con los objetivos previstos.

El trabajo cartográfico no está orientado a la generalización cartográfica, puesto que responde en un momento dado a unas necesidades y objetivos específicos que en la mayoría de los casos no consideran sistemas jerárquicos. Sin embargo, las necesidades posteriores de generalización son recurrentes y es ahí donde la organización jerárquica juega un papel fundamental para hacer de la generalización cartográfica una herramienta útil en la comprensión de los procesos que muestran propiedades emergentes o regularidades invariantes de los niveles jerárquicos representados en diferentes escalas. 
Con un modelo de organización jerárquica, la generalización parte de un alto nivel de resolución, representado en escala grande, al que cada vez se le disminuye un nivel jerárquico para mostrar un concepto cada vez más general en una escala cada vez más pequeña (figura 1). El proceso inverso no es posible, es decir, partir del nivel con más detalle, a una escala determinada, y representarlo a una escala mayor, puesto que instrumentalmente se requiere más información para lograrlo (figura 2).

Con el desarrollo de los procedimientos de generalización cartográfica para los ejemplos de cartografia temática, se vio la posibilidad de acelerar el proceso de generalización cartográfica en serie; por esto se recomienda programar los procedimientos de generalización cartográfica desarrollados (disolve, eliminate, simplify, centerline) y la asignación de las variables visuales según el contenido y la escala del mapa.

Se espera que el resultado de este trabajo, en los planos conceptual y aplicado, constituya un aporte académico en la formación de estudiantes y profesionales de diferentes disciplinas que se enfrentan a la representación de la organización espacial en diferentes niveles de resolución utilizando la herramienta cartográfica, tanto básica como temática. Este ejercicio que debe basarse en la concepción jerárquica del problema por representar cartográficamente en diferentes niveles de resolución.

\section{BIBLIOGRAFÍA}

Asamblea de Cundinamarca, Ordenanza 023 de 1998, por la cual se conforman las Provincias del Departamento de Cundinamarca, 1998.

Chadwick, G. A., Systems view of planning, segunda edición; Oxford; Pergamon Press, 1981.

Corporación Autónoma Regional, Boyacá, Pendientes. Plan de Ordenamiento Ambiental del Territorio Jurisdicción de Corpoboyacá. Escala 1:200.000. Bogotá, Corpoboyacá- UDFJC, 1997.

Departamento Administrativo del Medio Ambiente. Pendientes. Agenda Ambiental de la alcaldía local de Sumapaz D.C. Escala 1:50.000. Bogotá, DAMA, EEB UDFJC, 1994. 
Flórez, A., Generalización de las formas de ocupación del espacio: ejemplo teórico de clase en la asignatura Cartografía de la Maestría en Geografía, énfasis Ordenamiento Territorial, convenio UPTC-IGAC, 2004.

Flórez, A.; Montoya, J. W. “La generalización Cartográfica”, Revista Cartográfica 61. México, IPGH, 1992.

Haigh. M. J., "The Holon: Hierarchy theory and landscape research". Oxford, CATENA Suppl. 10: 181-192, 1987. Trad. A. Flórez, Cuadernos de Geografía. Revista del Departamento de Geografía de la Universidad Nacional de Colombia, Vol. V., No. 2, 1995.

Ibáñez, J. J., "Jerarquías en el sistema de suelos: la teoría de sistemas jerárquicos y los problemas escalares," Tendencias Científicas, Revista electrónica de ciencia, tecnología, sociedad y cultura. www.tendencias21. net. Febrero de 2006.

Instituto Geográfico Agustín Codazzi. Principios básicos de cartografía temática, Bogotá, D. C.; Ministerio de Hacienda y Crédito Público, IGAC, 1998.

International Cartographic Association (ICA), Multilingual Dictionary of Technical Terms in Cartography, Wiesbaden, GMBH, 1973.

Londoño, R.; Montaño, L., Pinto, D.; Rodríguez G., Estructura Urbano Regional Colombiana; Análisis Geográficos 17, Instituto Geográfico Agustín Codazzi, Bogotá, IGAC, 1986.

Torres C. J.; Lambert P., Join; "Diagnóstico Geográfico Nororiente del Vichada. Proyecto de Investigación en la Orinoquía. Programa Segunda Expedición Botánica”.Geomorfología, Vol. 6. Bogotá, IGAC, 1987.

Skarstein, A., "Generalization of Small Topographic Maps", in Contributions to Map Generalization Proceedings, Oslo, Harald Opheim Editor, 1980. 